RU-93-16

WIS-93/37/Apr-PH

April, 1993

\title{
Should Squarks Be Degenerate?
}

\author{
YOSEF NIR \\ Weizmann Institute of Science \\ Physics Department, Rehovot 76100, Israel \\ ftnir@weizmann.bitnet \\ and \\ Nathan SeIBerg \\ Department of Physics and Astronomy \\ Rutgers University, Piscataway, NJ 08855-0849, USA \\ seiberg@physics.rutgers.edu
}

\begin{abstract}
For generic squark masses, box diagrams with squarks and gluinos give unacceptably large contributions to neutral meson $(K, B$ and $D)$ mixing. The standard solution to this problem is to assume that squarks are degenerate to a very good approximation. We suggest an alternative mechanism to suppress squark contributions to flavor changing neutral currents: the alignment of quark with squark mass matrices. This mechanism arises naturally in the framework of Abelian horizontal symmetries.
\end{abstract}


Within the Standard Model, flavor changing neutral current (FCNC) interactions are highly suppressed by the weak coupling constant, by small mixing angles and by small fermion masses. This makes such processes a particularly sensitive probe of new physics at high energy scales. New contributions to FCNC may be comparable to or even dominate over the Standard Model contributions. Thus, measurements of FCNC processes such as neutral meson mixing [1],

$$
\begin{aligned}
& \frac{\Delta m_{K}}{m_{K}}=7 \times 10^{-15}, \\
& \frac{\Delta m_{B}}{m_{B}} \approx 7 \times 10^{-14}, \\
& \frac{\Delta m_{D}}{m_{D}} \leq 7 \times 10^{-14},
\end{aligned}
$$

and radiative $B$-decay [2],

$$
B R\left(B \rightarrow X_{s} \gamma\right) \leq 5.4 \times 10^{-4},
$$

put severe constraints on extensions of the Standard Model.

Supersymmetric extensions of the Standard Model predict large new contributions to FCNC processes. Squarks and gluinos contribute to (1) through box diagrams and to (2) through penguin diagrams. A possible suppression due to large squark and gluino masses is easily compensated for by three enhancement factors:

(i) Matrix elements of new four quark operators are enhanced due to different Lorentz structure;

(ii) The weak coupling of the Standard Model diagrams is replaced by the strong coupling;

(iii) The GIM mechanism does not operate for generic squark masses.

The resulting contributions are so large, even for squark masses as heavy as $1 \mathrm{TeV}$, that the processes (1) and (2) severely constrain the form of the squark mass matrices. 
A convenient way to present these constraints is the following. We denote the quark mass matrices and squark mass-squared matrices by $M^{q}$ and $\tilde{M}^{q 2}$, respectively $(q=u, d)$. Supersymmetry correlates the bases of the quarks and the squarks but in general there is no preferred basis for these superfields. However, as we will see below, models with horizontal symmetry have such a preferred basis. The $M^{q}$ matrices are diagonalized by bi-unitary transformations:

$$
\begin{aligned}
& V_{L}^{u} M^{u} V_{R}^{u \dagger}=\operatorname{diag}\left\{m_{u}, m_{c}, m_{t}\right\}, \\
& V_{L}^{d} M^{d} V_{R}^{d \dagger}=\operatorname{diag}\left\{m_{d}, m_{s}, m_{b}\right\} .
\end{aligned}
$$

The $6 \times 6$ matrices $\tilde{M}^{q 2}$ can be divided into $3 \times 3$ sub-matrices:

$$
\tilde{M}^{q 2}=\left(\begin{array}{cc}
\tilde{M}_{L L}^{q 2} & \tilde{M}_{L R}^{q 2} \\
\tilde{M}_{L R}^{q 2 \dagger} & \tilde{M}_{R R}^{q 2}
\end{array}\right)
$$

where $\tilde{M}_{L L}^{q 2}$ and $\tilde{M}_{R R}^{q 2}$ are hermitian matrices.

If all off-diagonal terms in $\tilde{M}^{q 2}$ are smaller than the diagonal ones, and $V_{L, R}^{q}$ are close to the identity, then the quantities constrained by FCNC processes are

$$
\left(\delta_{M N}^{q}\right)_{i j}=\frac{\left(V_{M}^{q} \tilde{M}_{M N}^{q 2} V_{N}^{q \dagger}\right)_{i j}}{\tilde{m}^{2}}
$$

(here and below $M$ and $N$ run over $L$ and $R$ ). The dimensionless $\delta_{M N}^{q}$-matrices have the simple meaning of squark mass-squared matrices (normalized to the average squark mass-squared $\tilde{m}^{2}$ ) in the basis where gluino couplings are diagonal and quark mass matrices are diagonal. The bounds are particularly strong on the combination

$$
\left\langle\delta_{i j}^{q}\right\rangle \equiv \sqrt{\left(\delta_{L L}^{q}\right)_{i j}\left(\delta_{R R}^{q}\right)_{i j}} .
$$

Using formulae from ref. [3] we present the constraints from eqs. (1) and (2) in Table 1. 
Table 1

Upper bounds on squark mass parameters from FCNC

\begin{tabular}{|c|c|c|c|c|c|c|c|c|c|}
\hline$\left\langle\delta_{12}^{d}\right\rangle$ & $\left(\delta_{M M}^{d}\right)_{12}$ & $\left(\delta_{L R}^{d}\right)_{12}$ & $\left\langle\delta_{13}^{d}\right\rangle$ & $\left(\delta_{M M}^{d}\right)_{13}$ & $\left(\delta_{L R}^{d}\right)_{13}$ & $\left(\delta_{L R}^{d}\right)_{23}$ & $\left\langle\delta_{12}^{u}\right\rangle$ & $\left(\delta_{M M}^{u}\right)_{12}$ & $\left(\delta_{L R}^{u}\right)_{12}$ \\
\hline 0.006 & 0.05 & 0.008 & 0.04 & 0.1 & 0.06 & 0.04 & 0.04 & 0.1 & 0.06 \\
\hline
\end{tabular}

The bounds in Table 1 correspond to $\tilde{m}=1 \mathrm{TeV}$ and scale like $\tilde{m}$. They are given for a gluino mass $m_{\tilde{g}}=\tilde{m}$, and become somewhat weaker (up to a factor of a few) for $m_{\tilde{g}}>\tilde{m}$. The matrix elements were calculated in the vacuum insertion approximation and we used $f_{B}=f_{D}=0.2 \mathrm{GeV}$ (the bounds on $\left(\delta_{M N}^{d}\right)_{13}$ and $\left(\delta_{M N}^{u}\right)_{12}$ scale like $1 / f_{B}$ and $1 / f_{D}$, respectively). The hadronic uncertainties (and the dependence on $\tilde{m}$ and on $\frac{m_{\tilde{g}}}{\tilde{m}}$ ) imply that the bounds in Table 1 should be trusted only to within a factor of, say, 3-4.

Bounds on $\left(\delta_{L L}^{d}\right)_{23},\left(\delta_{R R}^{d}\right)_{23}$ from $b \rightarrow s \gamma$ scale like $\tilde{m}^{2}$. At $\tilde{m} \sim 1 \mathrm{TeV}$, the suppression of the squark-gluino electromagnetic penguin diagram due to the heavy squark mass is strong enough to give no constraint on $\left(\delta_{M M}^{d}\right)_{23}$.

The bounds in Table 1 pose a serious problem to generic SUSY models, where $\left(\delta_{L L}^{q}\right)_{i j},\left(\delta_{R R}^{q}\right)_{i j}$, are expected to be of order one, and $\left(\delta_{L R}^{q}\right)_{i j}$ is expected to be of order $\frac{m_{Z}}{\tilde{m}}$. The standard solution to this problem is to assume

(a) Degeneracy (universality): each of the diagonal blocks, $\tilde{M}_{L L}^{q 2}$ and $\tilde{M}_{R R}^{q 2}$, is proportional to the unit matrix to a very good approximation;

(b) Proportionality: each of the nondiagonal blocks, $\tilde{M}_{L R}^{q 2}$, is proportional to the corresponding quark mass matrix $M^{q}$ to a very good approximation.

If the conditions of degeneracy and proportionality are fulfilled exactly, then the various $\delta_{M N}^{q}$ are diagonal and there is no contribution to FCNC processes. If the two conditions hold at some high energy scale but are violated by radiative corrections (as assumed in the minimal supersymmetric Standard Model), then a GIM-like suppression keeps the contributions within bounds.

These conditions have been known for a while (see e.g. [4]), and were discussed 
by numerous authors, including [5]. In the context of hidden sector supergravity models they were addressed by [6] but in the generic such model, they are unmotivated. This is also the case in string theory [7]. Both degeneracy and proportionality can be natural if SUSY breaking is communicated to the light particles by gauge interactions [8] or in models with a nonabelian horizontal symmetry [9].

In this work, we would like to suggest an alternative mechanism to suppress the squark contributions to FCNC: an approximate alignment of quark and squark mass matrices. We would first present the mechanism and then show that it arises naturally in the framework of Abelian horizontal symmetries.

The idea of quark-squark alignment is rather simple: assume that for some symmetry reason the matrices $\delta^{q}$ of eq. (5) (the squark mass-square matrices in the basis related by supersymmetry to the basis in which the quark mass matrices are diagonal) are diagonal. Then, regardless of whether the squarks are degenerate or not, squark contributions to FCNC vanish. In reality, we do not expect such an exact condition to hold, but it could naturally be a good approximation that would suppress FCNC to acceptable values.

The implementation of the quark-squark alignment mechanism for the offdiagonal blocks $\delta_{L R}^{q}$ is rather simple. If a symmetry leads to small entries in the quark mass matrices, it will at the same time lead to small entries in $\tilde{M}_{L R}^{q 2}$. In addition, a separation of the SUSY scale $\tilde{m}$ and the electroweak scale $m_{Z}$ will give a further suppression factor, $\frac{m_{Z}}{\tilde{m}}$. Together, the two factors typically give $\left(\delta_{L R}^{q}\right)_{i j} \sim \frac{\sqrt{m_{i}^{q} m_{j}^{q}}}{\tilde{m}}$, leading to very small contributions to FCNC.

To see explicitly how the approximate alignment works in the diagonal blocks, we neglect the small left-right mixing due to the nondiagonal blocks. (This approximation is well-justified in all models discussed below.) The $\tilde{M}_{L L}^{q 2}$ and $\tilde{M}_{R R}^{q 2}$ matrices are diagonalized by unitary transformations:

$$
\begin{aligned}
& \tilde{V}_{L}^{q} \tilde{M}_{L}^{q 2} \tilde{V}_{L}^{q \dagger}=\operatorname{diag}\left\{\tilde{m}_{q_{L 1}}^{2}, \tilde{m}_{q_{L 2}}^{2}, \tilde{m}_{q_{L 3}}^{2}\right\}, \\
& \tilde{V}_{R}^{q} \tilde{M}_{R}^{q 2} \tilde{V}_{R}^{q \dagger}=\operatorname{diag}\left\{\tilde{m}_{q_{R 1}}^{2}, \tilde{m}_{q_{R 2}}^{2}, \tilde{m}_{q_{R 3}}^{2}\right\} .
\end{aligned}
$$


In the basis where mass matrices for both quarks and squarks are diagonal, gluino interactions depend on mixing matrices $K_{L, R}^{q}$ :

$$
K_{L}^{q}=V_{L}^{q} \tilde{V}_{L}^{q \dagger}, \quad K_{R}^{q}=V_{R}^{q} \tilde{V}_{R}^{q \dagger} .
$$

The dependence of FCNC processes on squark masses and mixing is of the form

$$
\sum_{\alpha, \beta}\left(K_{M}^{q}\right)_{i \alpha}\left(K_{M}^{q}\right)_{j \alpha}^{*}\left(K_{N}^{q}\right)_{i \beta}\left(K_{N}^{q}\right)_{j \beta}^{*} f\left(\tilde{m}_{q_{M \alpha}}^{2}, \tilde{m}_{q_{N \beta}}^{2}\right),
$$

where $\alpha$ and $\beta$ label the squarks in the loop and $f\left(\tilde{m}_{q_{M \alpha}}^{2}, \tilde{m}_{q_{N \beta}}^{2}\right)$ is a function of squark masses.

There are two ways to suppress the off-diagonal terms $(i \neq j)$ in eq. (9):

1. The squarks in each sector are degenerate. Then, $f$ is independent of $\alpha$ and $\beta$ and the sum in eq. (9) simplifies,

$$
\sum_{\alpha}\left(K_{M}^{q}\right)_{i \alpha}\left(K_{M}^{q}\right)_{j \alpha}^{*}=0 \text { for } i \neq j
$$

where we used the unitarity of the $K$-matrices (equivalently, in this case $\tilde{V}^{q}$ are arbitrary). This is the degeneracy condition discussed above.

2. The matrices $K_{M}^{q}$ are close to the unit matrix,

$$
\left(K_{M}^{q}\right)_{i j} \ll 1 \text { for } i \neq j \text {. }
$$

Notice that for (11) to be fulfilled, the diagonalizing matrices for quarks, $V_{L, R}^{q}$, and the diagonalizing matrices for squarks, $\tilde{V}_{L, R}^{q}$, have to be approximately equal,

$$
V_{L}^{q} \tilde{V}_{L}^{q \dagger} \approx 1, \quad V_{R}^{q} \tilde{V}_{R}^{q \dagger} \approx 1, \quad(q=u, d) .
$$

Thus, it should be possible to simultaneously diagonalize (at least approximately) the quark mass matrices and the squark mass-squared matrices while preserving diagonal gluino interactions (i.e. the diagonalizing matrices act on the quark superfields). This is the alignment mechanism. 
If the squark masses are of the same order of magnitude, $\tilde{m}$, but not degenerate, the expression (9) is approximately

$$
\left(\max _{\alpha}\left(K_{M}^{q}\right)_{i \alpha}\left(K_{M}^{q}\right)_{j \alpha}^{*}\right)\left(\max _{\beta}\left(K_{N}^{q}\right)_{i \beta}\left(K_{N}^{q}\right)_{j \beta}^{*}\right) f\left(\tilde{m}^{2}, \tilde{m}^{2}\right)
$$

It is easy to see that the matrices $\delta_{M M}^{q}$ in eq. (5) are related to the matrices $K$ through

$$
\left(\delta_{M M}^{q}\right)_{i j} \sim \max _{\alpha}\left(K_{M}^{q}\right)_{i \alpha}\left(K_{M}^{q}\right)_{j \alpha}^{*}
$$

This estimate does not depend on assumptions such as the smallness of off-diagonal mass terms and therefore also on the smallness of mixing angles.

The constraints that arise from $K-\bar{K}$ mixing are the most difficult to satisfy. They require a particularly precise alignment in the first two generations of the down sector, namely $\left(K_{M}^{d}\right)_{12}$ have to be much smaller than the Cabibbo angle. This can be ensured if in some basis $M_{1 i}^{d}, M_{i 1}^{d}$ and $\left(\tilde{M}_{M M}^{d 2}\right)_{1 i}$ for $i=2,3$ are sufficiently small. $\quad M_{12}^{u}$ should then be sufficiently large in the same basis to produce the Cabibbo mixing. These conditions guarantee the alignment in other bases. In general there is no preferred basis and therefore such an alignment is not natural.

Quark-squark alignment arises naturally in models based on Abelian horizontal symmetry. First, such models have a natural basis for the quarks in which the alignment is as described in the previous paragraph. Second, they can naturally explain the hierarchy in the entries and can thus provide the necessary small numbers. Examples of such models were first suggested in ref. [10]. They have been extensively investigated in the context of grand unified models in ref. [11] and have recently been studied in the context of low energy supersymmetry in ref. [12]. In this framework the hierarchy in the quark sector parameters arises from a spontaneously broken horizontal discrete symmetry. If the symmetry were exact, only the top quark (and maybe the bottom quark) would have a mass. However, the symmetry is spontaneously broken by the $\operatorname{VEV}\langle S\rangle$ of a Standard Model singlet scalar. This breaking gives masses and mixings to all other quarks (except, maybe, 
the up quark) through nonrenormalizable terms, induced by integrating out heavy color-triplet fermions of mass $M>\langle S\rangle$. The hierarchy in mass ratios and mixing angles arises from their dependence on various powers of the ratio $\epsilon=\frac{\langle S\rangle}{M}$.

We now give explicit examples of models within this framework with precise enough alignment. The models are very similar to the ones presented in [12]. In all of these models, the horizontal symmetry is a discrete subgroup of

$$
H=U(1)_{X} \times U(1)_{H_{1}} \times U(1)_{H_{2}} .
$$

The $X$ charge of $\phi_{d}$ is -1 , that of all $\bar{d}_{i}$ is +1 and all other fields have vanishing $X$ charge. The scalar sector consists, in addition to the Standard Model doublets $\phi_{u}$ and $\phi_{d}$, of two Standard Model singlets $S_{1}$ and $S_{2}$. The $\left(H_{1}, H_{2}\right)$ charges of the scalars are

$$
\phi_{u}(0,0), \quad \phi_{d}(0,0), \quad S_{1}(-1,0), \quad S_{2}(0,-1)
$$

with the horizontal symmetry breaking parameters

$$
\epsilon_{1}=\frac{\left\langle S_{1}\right\rangle}{M} \sim 0.04, \quad \epsilon_{2}=\frac{\left\langle S_{2}\right\rangle}{M} \sim 0.008
$$

Here $M$ is a scale higher than the scale of symmetry breaking, $\left\langle S_{i}\right\rangle$, which communicates the breaking to the light fermions. The small parameters $\epsilon_{1}$ and $\epsilon_{2}$ determine the hierarchy in the quark sector parameters. Defining another small parameter $\epsilon \sim 0.2$, so that $\epsilon_{1} \sim \epsilon^{2} \sim 0.04$ and $\epsilon_{2} \sim \epsilon^{3} \sim 0.008$, we require that our models give

$$
\begin{aligned}
\epsilon & \sim\left|V_{u s}\right|, \\
\epsilon^{2} & \sim\left|V_{c b}\right|, \frac{m_{d}}{m_{s}}, \frac{m_{s}}{m_{b}}, \frac{m_{b}}{m_{t}}, \\
\epsilon^{3} & \sim\left|V_{u b}\right|, \frac{m_{u}}{m_{c}}, \frac{m_{c}}{m_{t}} .
\end{aligned}
$$

(An interesting option is to have $m_{u}=0$. This solves the strong CP problem but the phenomenological viability of this option is controversial. In this paper we choose to study only models that give $m_{u} \sim m_{d}$.) 
The horizontal charges of the second and third generation are uniquely fixed by eq. (18), but there are two possible $\left(H_{1}, H_{2}\right)$ assignments for each of $Q_{1}, \bar{u}_{1}$ and $\bar{d}_{1}$. It is on this point that our models differ from the one explicitly presented in ref. [12]. Requiring the alignment discussed above, the horizontal charges $\left(H_{1}, H_{2}\right)$ for $Q_{1}$ and $\bar{d}_{1}$ are fixed and we find two examples:

$$
\begin{array}{ccc}
Q_{1}(3,-1), & Q_{2}(1,0), & Q_{3}(0,0), \\
\bar{u}_{1}\left(h_{1}, h_{2}\right), & \bar{u}_{2}(-1,1), & \bar{u}_{3}(0,0), \\
\bar{d}_{1}(-3,3), & \bar{d}_{2}(1,0), & \bar{d}_{3}(1,0),
\end{array}
$$

with

$$
\left(h_{1}, h_{2}\right)= \begin{cases}(-3,3) & \text { Model A } \\ (0,1) & \text { Model B }\end{cases}
$$

The horizontal charges (19) lead to the following estimates for the various entries in the quark mass matrices:

$$
\begin{gathered}
\frac{M^{u}(\text { Model A })}{\left\langle\phi_{u}\right\rangle} \sim\left(\begin{array}{ccc}
\epsilon_{2}{ }^{2} & \epsilon_{1}{ }^{2} & 0 \\
0 & \epsilon_{2} & \epsilon_{1} \\
0 & 0 & 1
\end{array}\right), \frac{M^{u}(\text { Model B })}{\left\langle\phi_{u}\right\rangle} \sim\left(\begin{array}{ccc}
\epsilon_{1}{ }^{3} & \epsilon_{1}{ }^{2} & 0 \\
\epsilon_{1} \epsilon_{2} & \epsilon_{2} & \epsilon_{1} \\
\epsilon_{2} & 0 & 1
\end{array}\right), \\
\frac{M^{d}}{\left\langle\phi_{d}\right\rangle} \sim\left(\begin{array}{ccc}
\epsilon_{2}{ }^{2} & 0 & 0 \\
0 & \epsilon_{1}{ }^{2} & \epsilon_{1}{ }^{2} \\
0 & \epsilon_{1} & \epsilon_{1}
\end{array}\right) .
\end{gathered}
$$

The vanishing entries arise because in supersymmetric theories the Yukawa couplings are analytic in the scalars (we will return to this below). It is easy to check that the mass matrices (21) are consistent with (18). As in the model in [12] we explain eight small dimensionless numbers in terms of two small numbers, $\epsilon_{i}$, and thus obtain six order of magnitude mass-angle relations. However, without imposing $\epsilon_{1}^{3} \sim \epsilon_{2}^{2}$ in the present model we lose the celebrated relation $\theta_{c} \cong \sqrt{\frac{m_{d}}{m_{s}}}$ even as an order of magnitude one. 
An order of magnitude estimate of the various entries in the diagonal blocks of the squark mass-squared matrices gives

$$
\begin{aligned}
& \frac{\tilde{M}_{L L}^{2}}{\tilde{m}^{2}} \sim\left(\begin{array}{ccc}
1 & \epsilon_{1}{ }^{2} \epsilon_{2} & \epsilon_{1}{ }^{3} \epsilon_{2} \\
\epsilon_{1}{ }^{2} \epsilon_{2} & 1 & \epsilon_{1} \\
\epsilon_{1}{ }^{3} \epsilon_{2} & \epsilon_{1} & 1
\end{array}\right), \\
& \frac{\tilde{M}_{R R}^{u 2}}{\tilde{m}^{2}} \sim\left(\begin{array}{ccc}
1 & \epsilon_{1}{ }^{\left|h_{1}+1\right|} \epsilon_{2}{ }^{\left|h_{2}-1\right|} & \epsilon_{1}{ }^{\left|h_{1}\right|} \epsilon_{2}{ }^{\left|h_{2}\right|} \\
\epsilon_{1}{ }^{\left|h_{1}+1\right|} \epsilon_{2}{ }^{\left|h_{2}-1\right|} & 1 & \epsilon_{1} \epsilon_{2} \\
\epsilon_{1}{ }^{\left|h_{1}\right|} \epsilon_{2}{ }^{\left|h_{2}\right|} & \epsilon_{1} \epsilon_{2} & 1
\end{array}\right), \\
& \frac{\tilde{M}_{R R}^{d 2}}{\tilde{m}^{2}} \sim\left(\begin{array}{ccc}
1 & \epsilon_{1}{ }^{4} \epsilon_{2}{ }^{3} & \epsilon_{1}{ }^{4} \epsilon_{2}{ }^{3} \\
\epsilon_{1}{ }^{4} \epsilon_{2}{ }^{3} & 1 & 1 \\
\epsilon_{1}{ }^{4} \epsilon_{2}{ }^{3} & 1 & 1
\end{array}\right)
\end{aligned}
$$

(unlike the quarks mass matrices and the nondiagonal blocks, these do not have to be analytic in the $S$ fields). For the nondiagonal blocks,

$$
\frac{\left(\tilde{M}_{L R}^{q 2}\right)_{i j}}{\tilde{m}} \sim\left(M^{q}\right)_{i j}
$$

where the $\sim$ sign indicates that the different entries are of the same order of magnitude.

With the above mass matrices, it is possible to estimate the various $\left(\delta_{M N}^{q}\right)_{i j}$. As argued above, the $\left(\delta_{L R}^{q}\right)_{i j}$ contributions are very small. To estimate $\left(\delta_{M M}^{q}\right)_{i j}$ we note that in our framework all squark masses are of order $\tilde{m}$ but not degenerate. Then eq. (14) holds. Taking into account that all diagonal elements in the diagonalizing matrices are of order one, we find

$$
\begin{aligned}
\left(\delta_{L L}^{d}\right)_{12} \sim \max \{ & \left(V_{L}^{d}\right)_{12}, \quad\left(\tilde{V}_{L}^{d}\right)_{12},\left(V_{L}^{d}\right)_{13}\left(\tilde{V}_{L}^{d}\right)_{23}, \\
& \left.\left(V_{L}^{d}\right)_{23}\left(\tilde{V}_{L}^{d}\right)_{13},\left(V_{L}^{d}\right)_{13}\left(V_{L}^{d}\right)_{23}, \quad\left(\tilde{V}_{L}^{d}\right)_{13}\left(\tilde{V}_{L}^{d}\right)_{23}\right\}
\end{aligned}
$$

and similarly for the other $\left(\delta_{M M}^{q}\right)_{i j}$. 
Our results are summarized in Tables 2 and 3. We give here the predictions of the two models discussed above - model A and model B - for the various $\left(\delta_{M M}^{q}\right)_{i j}$ relevant to FCNC. These predictions are compared to the phenomenological constraints of Table 1 . As the constraints on $\left(\delta_{L R}^{q}\right)_{i j}$ (and on $\left.\left(\delta_{M M}^{d}\right)_{23}\right)$ are fulfilled almost trivially in our framework, we do not present them in the Tables. Table 2 presents the predictions for the down sector, which are identical for both models. The predictions for $D-\bar{D}$ mixing, presented in Table 3 , are different in the two models, but in both they are close to the experimental bounds.

Table 2

SUSY contributions to down sector FCNC

\begin{tabular}{|c|c|c|c|c|c|c|}
\hline & $\left\langle\delta_{12}^{d}\right\rangle$ & $\left(\delta_{L L}^{d}\right)_{12}$ & $\left(\delta_{R R}^{d}\right)_{12}$ & $\left\langle\delta_{13}^{d}\right\rangle$ & $\left(\delta_{L L}^{d}\right)_{13}$ & $\left(\delta_{R R}^{d}\right)_{13}$ \\
\hline Expt. Upper Bound & 0.006 & 0.05 & 0.05 & 0.04 & 0.1 & 0.1 \\
\hline Models A, B & $\epsilon_{1}{ }^{3} \epsilon_{2}{ }^{2}$ & $\epsilon_{1}{ }^{2} \epsilon_{2}$ & $\epsilon_{1}{ }^{4} \epsilon_{2}{ }^{3}$ & $\epsilon_{1}{ }^{7 / 2} \epsilon_{2}{ }^{2}$ & $\epsilon_{1}{ }^{3} \epsilon_{2}$ & $\epsilon_{1}^{4} \epsilon_{2}{ }^{3}$ \\
& $\sim 4 \cdot 10^{-9}$ & $\sim 10^{-5}$ & $\sim 10^{-12}$ & $\sim 10^{-9}$ & $\sim 5 \cdot 10^{-7}$ & $\sim 10^{-12}$ \\
\hline
\end{tabular}

Table 3

SUSY contributions to up sector FCNC

\begin{tabular}{|c|c|c|c|}
\hline & $\left\langle\delta_{12}^{u}\right\rangle$ & $\left(\delta_{L L}^{u}\right)_{12}$ & $\left(\delta_{R R}^{u}\right)_{12}$ \\
\hline Expt. Upper Bound & 0.04 & 0.1 & 0.1 \\
\hline Model A & $\epsilon_{1}^{2} / \epsilon_{2}^{1 / 2}$ & $\epsilon_{1}^{2} / \epsilon_{2}$ & $\epsilon_{1}^{2}$ \\
& $\sim 0.02$ & $\sim 0.2$ & $\sim 10^{-3}$ \\
\hline Model B & $\epsilon_{1}^{3 / 2} / \epsilon_{2}^{1 / 2}$ & $\epsilon_{1}^{2} / \epsilon_{2}$ & $\epsilon_{1}$ \\
& $\sim 0.09$ & $\sim 0.2$ & $\sim 0.04$ \\
\hline
\end{tabular}

We conclude that these two examples satisfy all the constraints from FCNC without any degeneracy among squarks. (Remember that both the experimental 
upper bound and the order of magnitude predictions in our models suffer from ambiguities in multiplicative factors of order one.)

As discussed above, the most difficult condition to satisfy is

$$
\left(V_{L}^{d}\right)_{12}, \quad\left(V_{R}^{d}\right)_{12} \lesssim 0.006
$$

which comes from $K-\bar{K}$ mixing. It is naturally guaranteed in our models because the horizontal symmetry forces $M_{1 i}^{d}=0$ and $M_{i 1}^{d}=0$ for $i=2,3$. Such vanishing entries arise because of a combination of the horizontal symmetry and the analyticity of the superpotential. Only terms of the form $Q_{i} \phi_{d} \bar{d}_{j}\left(\frac{S_{1}}{M}\right)^{m}\left(\frac{S_{2}}{M}\right)^{n}$ (with non-negative $n$ and $m$ ) are supersymmetric but for these entries such terms are not compatible with the horizontal symmetry (e.g. $\left.H_{2}\left(Q_{1}\right)+H_{2}\left(\bar{d}_{2}\right)<0\right)$. We will see below that soft SUSY breaking can generate nonanalytic terms, but they are suppressed at least by several powers of $\epsilon$ 's as dictated by the horizontal symmetry (e.g. $M_{12}^{d} \sim \epsilon_{1}^{4} \epsilon_{2} \sim 2 \cdot 10^{-8}$ ) which render them harmless.

The entries in the quark mass matrices can be modified by several mechanisms:

(i) The soft SUSY breaking terms can introduce non-analytic Yukawa terms with $S_{i}^{\dagger}$. These can arise either at tree level by integrating out the fields at the scale $M$ and are suppressed by $\frac{m_{3 / 2}}{M}$ or by loops in the low energy theory in which case they are suppressed by at least $\frac{\alpha_{s}}{\pi}$. As mentioned in the previous paragraph, the suppression by factors of $\epsilon$ 's makes these very small.

(ii) The full Lagrangian has only a discrete horizontal symmetry, say $Z_{n}$. Then, for non negative $m$ such that $H\left(Q_{1}\right)+H\left(\bar{d}_{2}\right)+m H\left(S_{i}\right)=-n$, the term $Q_{1} \phi_{d} \bar{d}_{2}\left(\frac{S_{i}}{M}\right)^{m}$ is $Z_{n}$-invariant. We have to check then that $\epsilon_{i}^{m}$ is small enough to make this contribution harmless.

(iii) The group $U(1)_{X}$ must be broken because otherwise the model has an unacceptable axion at the weak scale. This introduces factors of $\eta^{2}=\frac{\left\langle\phi_{u}\right\rangle\left\langle\phi_{d}\right\rangle}{M^{2}}$ into the mass matrices. For sufficiently large $M$, the effects of these factors on tree level FCNC, on the hierarchy and on the alignment are negligible ${ }^{\star}$.

^ We thank M. Leurer for a useful discussion on this point. 
(iv) In some specific high-energy models, some terms allowed by all symmetries are not generated by integrating out the massive fields at tree level. This can happen if there are too few massive particles to induce all transitions [12]. We have to check that all necessary terms can indeed be generated.

The high energy spectrum needed in our models consists of at least four massive fermions of charge $+2 / 3$ and five of charge $-1 / 3$. To avoid Landau poles below the Planck scale, we need either $M \gtrsim 10^{7} \mathrm{TeV}$ or a larger symmetry group at high energies [12].

We have constructed explicit models belonging to the class of models defined by eq. (19) but where $U(1)_{X}$ is broken in the superpotential by terms of the form $\phi_{u} \phi_{d} S_{i}$ or $\phi_{u} \phi_{d}$ and where only an anomaly-free discrete subgroup is maintained. Explicitly, in Model A (B), the anomaly free symmetry is $Z_{n} \times Z_{5}$ with $n \geq 3$ $\left(Z_{6} \times Z_{n}\right.$ with $\left.n \geq 3\right)$. In both models, the zeros in $M^{d}$ are lifted but the quarksquark alignment remains accurate enough to satisfy all constraints.

Additional constraints on squark parameters from FCNC arise from diagrams that involve winos ${ }^{\dagger}$. When $S U(2)$ breaking effects in the squark mass matrices are negligible - as is the case in our framework - the mixing angles for the couplings $\tilde{w}^{-} u_{i} \tilde{d}_{j}^{\dagger}$ and $\tilde{w}^{+} d_{i} \tilde{u}_{j}^{\dagger}$ are $\left(K_{L}^{u}\right)_{i j}$ and $\left(K_{L}^{d}\right)_{i j}$, respectively. Consequently, for $m_{\tilde{w}} \sim$ $m_{\tilde{g}}$, diagrams with intermediate winos are suppressed by $\left(\frac{g}{g_{s}}\right)^{4}$ compared to the LL gluino diagrams. The constraints from wino interactions are fulfilled whenever those from gluino interactions are. (A similar statement can be made on constraints from zino and photino interactions.)

To summarize: models with Abelian horizontal symmetries, originally constructed to explain the hierarchy in the quark sector parameters, can naturally align the mass matrices of quarks with the mass-squared matrices for squarks, to a good approximation. As a result, the contributions from squark-gluino loop diagrams to neutral meson mixing and to radiative $B$ decay are suppressed compared

$\dagger$ We thank M. Dine for a useful discussion on these diagrams. 
to a generic low-energy supersymmetric framework, even though there is no degeneracy among squarks. We would like to stress, however, that our main point is the alignment. The models based on horizontal symmetry are only examples meant to demonstrate that the alignment can be natural.

This class of models where the bounds from $K-\bar{K}$ mixing are satisfied have the special feature that the Cabibbo mixing between the first and second generation arises dominantly from mixing in the up sector. (Remember that here, unlike the Standard Model, we have a natural interaction basis, the one where the horizontal charges are diagonal.) This leads to a testable signature of the quark-squark alignment mechanism, namely that $D-\bar{D}$ mixing should be close to the experimental

upper bound. This is in contrast to the Standard Model, where [13] $\frac{\Delta m_{D}}{m_{D}} \sim 10^{-15}$, and maybe even considerably smaller than that if heavy quark symmetry considerations apply [14]. Of course, the most prominent prediction (although it will take some time to examine it experimentally) of our scheme is that, in contradiction with the conventional wisdom, the squarks are not degenerate.

\section{ACKNOWLEDGMENTS}

It is a pleasure to thank T. Banks, A. Dabholkar, M. Dine, K. Intriligator and M. Leurer for several useful discussions. We also thank T. Banks and M. Dine for comments on the manuscript. YN is grateful to the theory group at Rutgers University for their hospitality. YN is an incumbent of the Ruth E. Recu Career Development Chair and is supported, in part, by the Israel Commission for Basic Research and by the Minerva Foundation. This work was supported in part by DOE grant DE-FG05-90ER40559. 


\section{REFERENCES}

1. K. Hikasa et al., Particle Data Group, Phys. Rev. D45 (1992) S1.

2. E. Thorndike, CLEO Collaboration, a talk given in the meeting of the American Physical Society, Washington D.C. (1993).

3. F. Gabbiani and A. Masiero, Nucl. Phys. B322 (1989) 235.

4. H.P. Nilles, Phys. Rep. 110 (1984) 1.

5. H. Georgi, Phys. Lett. 169B (1986) 231; L.J. Hall, V.A. Kostelecky and S. Raby, Nucl. Phys. B267 (1986) 415.

6. L. Hall, J. Lykken and S. Weinberg, Phys. Rev. D27 (1983) 2359.

7. M. Dine, A. Kagan and S. Samuel, Phys. Lett. 243B (1990) 250; L. Ibanez and D. Lust, Nucl. Phys. B382 (1992) 305; B. de Carlos, J.A. Casas and C. Munoz, Phys. Lett. 299B (1993) 234; CERN-TH.6436/92; V. Kaplunovski and J. Louis, CERN-TH.6809/93 (1993).

8. M. Dine and A.E. Nelson, SCIPP 93/03 (1993).

9. M. Dine, A. Kagan and R. Leigh, SCIPP 93/04 (1993).

10. C.D. Froggatt and H.B. Nielsen, Nucl. Phys. B147 (1979) 277.

11. Z.G. Berezhiani, Phys. Lett. 129B (1983) 99; 150B (1985) 177; S. Dimopoulos, Phys. Lett. 129B (1983) 417; J. Bagger, S. Dimopoulos, E. Masso and M. Reno,Nucl. Phys. B258 (1985) 565; J. Bagger, S. Dimopoulos, H. Georgi and S. Raby, In: Proc. Fifth Workshop on Grand Unification. Eds. Kang, K., Fried, H. and Frampton, P., Singapore, World Scientific (1984); A. Davidson, V.P. Nair and K.C. Wali, Phys. Rev. D29 (1984) 1505; A. Davidson and

K.C. Wali, Phys. Rev. Lett. 60 (1988) 1313; A. Davidson, S. Ranfone and K.C. Wali, Phys. Rev. D41 (1990) 208.

12. M. Leurer, Y. Nir and N. Seiberg, RU-92/59, Nucl. Phys. B, in press.

13. J. F. Donoghue, E. Golowich, B. R. Holstein and J. Trampetic, Phys. Rev. D33 (1986) 179. 
14. H. Georgi, HUTP-92/A049 (1992); T. Ohl, G. Ricciardi, E.H. Simmons, HUTP-92/A053. 